\title{
Seletividade e EFicácia de Herbicidas Inibidores da Enzima accase na Cultura da mamona ${ }^{1}$
}

\author{
Selectivity and Efficacy of ACCase-Inhibiting Herbicides in Castor Bean Crop
MACIEL, C.D.G. ${ }^{2}$, SILVA, T.R.B. ${ }^{2}$, POLETINE, J.P. ${ }^{2}$, VELINI, E.D. ${ }^{3}$, ZANOTTO, M.D. ${ }^{3}$, MARTINS, F.M. ${ }^{4}$ e GAVA, F. ${ }^{4}$

\begin{abstract}
RESUMO - O experimento foi conduzido com o objetivo de avaliar a seletividade e eficácia de herbicidas inibidores de ACCase na cultura da mamoneira AL Guarany 2002, no municipio de Paraguaçu Paulista/SP, durante a safra 2002/2003. O delineamento experimental utilizado foi o de blocos ao acaso, com 11 tratamentos e quatro repetições, constituidos pelos herbicidas e adjuvantes: fluazifop-p-butil $\left(313 \mathrm{~g} \mathrm{ha}^{-1}\right)+\operatorname{Agral}^{\oplus}(0,2 \% \mathrm{v} / \mathrm{v})$; sethoxydim $\left(322 \mathrm{~g} \mathrm{ha}^{-1}\right)+$ Assist $^{\circledR}$ $(0,5 \% \mathrm{v} / \mathrm{v})$; haloxyfop-methyl $\left(120 \mathrm{~g} \mathrm{ha}^{-1}\right)+$ Assist $^{\circledR}(0,5 \% \mathrm{v} / \mathrm{v})$; clethodim+fenoxaprop-p-ethyl $\left(75 \mathrm{~g} \mathrm{ha}^{-1}\right)+\operatorname{Assist}^{\circledR}(0,5 \% \mathrm{v} / \mathrm{v})$; quizalofop-p-ethyl (125 g ha $\left.{ }^{1}\right)+$ Assist $^{\circledR}(0,5 \% \mathrm{v} / \mathrm{v})$; clethodim $\left(156 \mathrm{~g} \mathrm{ha}^{-1}\right)+\operatorname{Assist}^{\circledR}(0,5 \% \mathrm{v} / \mathrm{v})$; propaquizafop $\left(175 \mathrm{~g} \mathrm{ha}^{-1}\right)+\operatorname{Assist}^{\circledR}(0,5 \% \mathrm{v} / \mathrm{v})$; tepraloxydim $\left(400 \mathrm{~g} \mathrm{ha}^{-1}\right)+\operatorname{Dash}^{\circledast}(0,5 \% \mathrm{v} / \mathrm{v})$; butroxydim $\left(100 \mathrm{~g} \mathrm{ha}^{-1}\right)+\operatorname{Dash}^{\circledast}(0,5 \% \mathrm{v} / \mathrm{v})$; isoxaflutole (60 $\left.\mathrm{g} \mathrm{ha}^{-1}\right)$; e testemunha capinada. No momento da aplicação a mamoneira encontrava-se com 4 a 6 folhas, e o Cenchrus echinatus, com 1 a 5 perfilhos. A mamoneira cv. AL Guarany 2002 apresentou alta seletividade aos herbicidas inibidores de ACCase, não sendo verificada fitointoxicação aos 14 DAA (dias após aplicação), com exceção do tepraloxydim, onde os sintomas persistiram até os 21 DAA, e do isoxaflutole (inibidor de HPPD), por apresentar injúrias nas folhas mais velhas e redução significativa de produtividade. A infestação de C. echinatus foi eficientemente controlada pelos herbicidas inibidores de ACCase entre $14 \mathrm{e}$ 21 DAA $(\geq 95,0 \%)$.
\end{abstract}

Palavras-chave: Ricinus communis, fitointoxicação, controle, produtividade

\begin{abstract}
This experiment aimed to evaluate the selectivity and efficacy of ACCase-inhibiting herbicides in castor bean crop, at Paraguaçu Paulista, São Paulo, during the 2002/03 agricultural season. The experimental design used was randomized complete blocks, with eleven treatments and four replicates, constituted by the following herbicides and adjuvants: fluazifop-p-butyl (313 $\left.g h^{-1}\right)$ $+\operatorname{Agral}^{\text {TM }}(0.2 \% \mathrm{v} / \mathrm{v})$; sethoxydim $\left(322 \mathrm{~g} \mathrm{ha}^{-1}\right)+\operatorname{Assist}{ }^{T M}(0.5 \% \mathrm{v} / \mathrm{v})$; haloxyfop-methyl $\left(120 \mathrm{~g} \mathrm{ha} \mathrm{H}^{-1}\right)+$

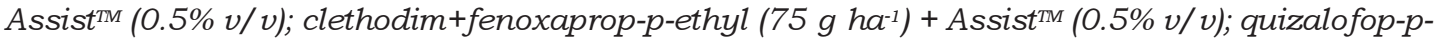
ethyl (125 $\left.\mathrm{g} \mathrm{ha}^{-1}\right)+$ Assist ${ }^{T M}(0.5 \% \mathrm{v} / \mathrm{v})$; clethodim (156 $\left.\mathrm{g} \mathrm{ha}^{-1}\right)+\operatorname{Assist}^{T M}(0.5 \% \mathrm{v} / \mathrm{v})$; propaquizafop $\left(175 \mathrm{~g} \mathrm{ha}^{-1}\right)+\operatorname{Assist}^{T M}(0.5 \% \mathrm{v} / \mathrm{v})$; tepraloxydim $\left(400 \mathrm{~g} \mathrm{ha}^{-1}\right)+\operatorname{Dash}^{T M}(0.5 \% \mathrm{v} / \mathrm{v})$; butroxydim $\left(100 \mathrm{~g} \mathrm{ha}^{-1}\right)+\operatorname{Dash}^{T M}(0.5 \% \mathrm{v} / \mathrm{v})$; isoxaflutole $\left(60 \mathrm{~g} \mathrm{ha}^{-1}\right)$ and weed-free check. At the moment of application, castor bean plants presented 4 to 6 leaves and Cenchrus echinatus, 1 to 5 tillers. $A L$ Guarany 2002 cultivar presented high selectivity to ACCase-inhibiting herbicides, without visible symptoms of phytotoxicity in 14 DAA (days after application), except fortepraloxydim, whose symptoms persisted until 21 DAA, and isoxaflutole (HPPD inhibiting), which showed injuries in older leaves and significant yield reduction. C. echinatus infestation was efficiently controlled by ACCase-inhibiting herbicides between 14 and 21 DAA $(\geq 95.0 \%)$.
\end{abstract}

Keywords: Ricinus communis, phytotoxicity, control, yield.

1 Recebido para publicação em 27.10.2010 e aprovado em 10.2.2011.

2 Engo-Agr ${ }^{\circ}$., D.Sc., Professor Adjunto, Dep. de Ciências Agronômicas, Universidade Estadual de Maringá - CCA/UEM, Campus Avançado de Umuarama-PR - CAU, Estrada da Paca s/n, 87507-190 Umuarama-PR, <cdgmaciel2@uem.br>; ${ }^{3}$ Engo-Agro${ }^{\circ}$, D.Sc., Professor Assistente, Faculdade de Ciência Agronômica, Universidade Estadual Paulista "Júlio de Mesquita Filho" - FCA/UNESP, Fazenda Lageado, Rua José Barbosa de Barros, 1780, 18610-307 Botucatu-SP, ${ }^{5}$ Acadêmicos do Curso de Agronomia, Escola Superior de Agronomia de Paraguaçu Paulista - FUNGE/ESAPP.

Planta Daninha, Viçosa-MG, v. 29, n. 3, p. 609-616, 2011 


\section{INTRODUÇÃO}

A mamoneira (Ricinus communis) é uma oleaginosa de relevante importância econômica e social, cujo óleo possui inúmeras aplicações na área industrial e perspectiva de utilização como fonte energética na produção de biocombustível (Severino, 2006). Atualmente, o Brasil é o terceiro maior produtor de mamona (FAO, 2009), com estimativa de produção de aproximadamente 101 mil toneladas na safra 2009/10, porém com produtividade média ainda baixa (CONAB, 2010)

Conhecida pela versatilidade de uso do óleo extraído de suas sementes, assim como pela rusticidade, a planta de mamoneira é capaz de se adaptar a diferentes condições ambientais e produzir satisfatoriamente até sob baixa precipitação pluvial. Mesmo tendo sua produtividade afetada, tem-se mostrado resistente ao clima adverso, ao passo que se verificam perdas totais em outras culturas, servindo como alternativa de trabalho e renda sobretudo para o pequeno agricultor (Melhorança \& Staut, 2005; Silva et al., 2007).

Apesar de sua importância, com relação ao nivel de tecnologia empregado na cultura, assim como as dimensões das propriedades e alguns aspectos culturais, como, por exemplo, o controle de plantas daninhas, são considerações ainda bastantes variáveis de região para região (Oliveira et al., 2005). Segundo Maciel et al. (2008), o uso de herbicidas na cultura da mamona, apesar de não ser o mais difundido entre os produtores, provavelmente, é o método de controle mais prático e econômico de manejo das plantas daninhas, principalmente para cultivos mais tecnificados de grandes áreas, como é o caso dos híbridos de mamoneira de porte anão. Com a redução do espaçamento entre plantas, em função da colheita mecanizada desses novos genótipos de porte baixo, o uso de herbicidas será ferramenta fundamental para viabilizar a mamoneira nesse sistema de produção intensiva. No entanto, prevalece o desinteresse das indústrias de defensivos no desenvolvimento de herbicidas seletivos, além da inexistência de estudos de técnicas de aplicação alternativas que viabilizem o controle satisfatório das plantas daninhas sem prejudicar a mamoneira, e poucas são as alternativas viáveis de controle químico.
Para o controle de plantas daninhas gramíneas na cultura da mamona, Maciel et al. (2007) mencionaram a seletividade dos cultivares Íris e AL Guarany 2002 aos herbicidas pendimethalin, alachlor+pendimethalin e alachlor+trifluralin em aplicações de préplantio incorporado e a clomazone e clomazone+trifluralin em pré-emergência da cultura. Entretanto, na literatura são praticamente inexistentes estudos que comprovem a viabilidade de herbicidas aplicados em pós-emergência da mamoneira. Também, bastante é conhecida pelos produtores a viabilidade de herbicidas exclusivamente graminicidas, ou seja, dos inibidores da enzima acetilCoA carboxilase (ACCase), os quais ainda não estão oficialmente registrados para aplicação em pós-emergência na cultura da mamona. Essa enzima catalisa a primeira reação química, a partir de acetil-CoA, para sintese de lipídeos nas células vegetais (Vidal \& Merotto Jr., 2001).

Este trabalho teve como objetivo avaliar a seletividade e eficiência de herbicidas inibidores da enzima ACCase, em aplicação de pósemergência da mamona variedade AL Guarany 2002, por meio da análise de características agronômicas culturais.

\section{MATERIAL E MÉTODOS}

O trabalho foi realizado em Paraguaçu Paulista/SP - FUNGE/ESAPP, em altitude média de $480 \mathrm{~m}$ (latitude de $22^{\circ} 24^{\prime} 46^{\prime \prime} \mathrm{S}$ e longitude de $\left.50^{\circ} 34^{\prime} 33^{\prime \prime} \mathrm{W}\right)$, no período de dezembro de 2002 a maio de 2003 . O clima da região, segundo classificação de Köppen, é do tipo Aw, caracterizado por savana com inverno seco. A temperatura média anual é de $22,8{ }^{\circ} \mathrm{C}$, com amplitude em torno de $5{ }^{\circ} \mathrm{C}$; a precipitação média anual é de $1.361 \mathrm{~mm}$, com período chuvoso de dezembro a fevereiro e seco de junho a agosto.

O solo da área experimental foi classificado como Latossolo Vermelho distrófico de textura arenosa, sendo constituído por $830 \mathrm{~g} \mathrm{~kg}^{-1} \mathrm{de}$ areia total, $120 \mathrm{~g} \mathrm{~kg}^{-1}$ de argila e $50 \mathrm{~g} \mathrm{~kg}^{-1}$ de silte; as características químicas amostradas antes da instalação do ensaio eram: $\mathrm{pH}\left(\mathrm{CaCl}_{2}\right)$ $=5,8 ; \mathrm{MO}=10,0 \mathrm{~g} \mathrm{dm}^{-3} ; \mathrm{P}=20,0 \mathrm{~g} \mathrm{dm}^{-3} ; \mathrm{K}^{+}=$ 2,$9 ; \mathrm{H}^{+}+\mathrm{Al}^{+3}=9,0, \mathrm{Ca}^{+2}=12,0 ; \mathrm{Mg}=5,0 ; \mathrm{CTC}=$ 29,$0 ;$ e V\% $=69$. 
A semeadura da variedade AL Guarany 2002 foi realizada manualmente em 14/12/ 2002, em solo classificado como Latossolo Vermelho distrófico de textura arenosa, utilizando-se espaçamento de 1,0 x 1,0 m entre linhas e plantas, três sementes por cova e posterior desbaste após a germinação. A adubação de semeadura foi realizada com a formulação NPK 04-14-08, na dosagem de $300 \mathrm{~kg} \mathrm{ha}^{-1}$; aos 25 dias após a emergência efetuou-se a adubação de cobertura, com $75 \mathrm{~kg} \mathrm{ha}^{-1}$ de ureia. Os demais tratos culturais, com exceção do controle de plantas daninhas, foram desenvolvidos conforme informações e recomendações técnicas para a cultura da mamona (Savy Filho, 2005).

O delineamento experimental foi o de blocos casualizados, com 11 tratamentos e quatro repetições; as unidades experimentais foram constituídas por parcelas de 3,0 x 6,0 m $\left(18,0 \mathrm{~m}^{2}\right)$, totalizando área experimental de $792,0 \mathrm{~m}^{2}$. Os tratamentos estudados foram constituídos pelos herbicidas e adjuvantes: fluazifop-p-butil (313 $\left.\mathrm{g} \mathrm{ha}^{-1}\right)+\operatorname{Agral}^{\circledast}(0,2 \% \mathrm{v} / \mathrm{v})$; sethoxydim $\left(322 \mathrm{~g} \mathrm{ha}^{-1}\right)+$ Assist $^{\circledR}(0,5 \% \mathrm{v} / \mathrm{v})$; haloxyfop-methyl $\left(120 \mathrm{~g} \mathrm{ha}^{-1}\right)+$ Assist $^{\circledR}(0,5 \%$ $\mathrm{v} / \mathrm{v})$; clethodim + fenoxaprop-p-ethyl $\left(75+75\right.$ g ha $\left.^{-1}\right)+$ Assist $^{\circledR}(0,5 \%$ v/v); quizalofopp-ethyl (125 g ha $\left.^{-1}\right)+$ Assist $^{\circledR}(0,5 \% \mathrm{v} / \mathrm{v})$; clethodim $\left(156 \mathrm{~g} \mathrm{ha}^{-1}\right)+$ Assist $^{\oplus}(0,5 \% \mathrm{v} / \mathrm{v})$; propaquizafop (175 $\left.\mathrm{g} \mathrm{ha}^{-1}\right)+$ Assist $^{\circledast}(0,5 \% \mathrm{v} / \mathrm{v})$; tepraloxydim $\left(400 \mathrm{~g} \mathrm{ha}^{-1}\right)+\operatorname{Dash}^{\circledast}(0,5 \% \mathrm{v} / \mathrm{v})$; butroxydim $\left(100 \mathrm{~g} \mathrm{ha}^{-1}\right)+\operatorname{Dash}^{\circledast}(0,5 \% \mathrm{v} / \mathrm{v})$; isoxaflutole $\left(60 \mathrm{~g} \mathrm{ha}^{-1}\right)$; e testemunha capinada. A fim de de verificar o limite máximo dos aspectos de seletividade da mamoneira, em todos os tratamentos com os inibidores de ACCase utilizaram-se doses superiores às registradas para culturas oleaginosas (Rodrigues \& Almeida, 2005), com destaque para os produtos haloxyfop-methyl (duas vezes a dose comercial) e tepraloxydim (quatro vezes a dose comercial).

A aplicação dos herbicidas foi realizada em pós-emergência em 18/1/2003, quando as plantas de mamoneira encontravam-se com 4 a 6 folhas e a infestação predominante de Cenchrus echinatus ( 15 plantas $\left.\mathrm{m}^{2}\right)$ situava-se entre 1 e 5 perfilhos. Na aplicação foi utilizado pulverizador costal pressurizado a $\mathrm{CO}_{2}$, com quatro pontas XR11002-VS, pressão de $210 \mathrm{kPa}$ e consumo de calda de $200 \mathrm{~L} \mathrm{ha}^{-1}$. No momento da aplicação o solo encontrava-se úmido e o céu sem nebulosidade; as condições climáticas registradas em anemômetro digital indicaram temperatura e umidade relativa do ar em $28,5{ }^{\circ} \mathrm{C}$ e $67,3 \%$, respectivamente, e velocidade dos ventos em torno de $2,0 \mathrm{~km} \mathrm{~h}^{-1}$.

As características agronômicas analisadas foram: fitointoxicação da cultura e controle da plantas daninhas aos 7, 14, 21 e 28 dias após aplicação (DAA), por meio de avaliação visual $(\%)$, conforme escala de notas proposta pela SBCPD (1995), em que 0\% correspondeu à ausência de injúrias e $100 \%$ à morte das plantas; teor de clorofila em folhas novas (terço superior) e velhas (terço inferior) aos $7 \mathrm{e}$ 21 DAA, utilizando clorofilômetro portátil modelo Minolta ${ }^{\circledR}\left(\mu \mathrm{g} \mathrm{dm}^{-2}\right)$; comprimento do caule $(\mathrm{cm})$ aos 7 e 21 DAA, medindo-se desde a superfície do solo até o início a inserção do racemo primário da planta; diâmetro mediano caulinar (mm) aos 7 e 21 DAA, aferido no primeiro entrenó das plantas; e produtividade de grãos $\left(\mathrm{kg} \mathrm{ha}^{-1}\right)$ aos 130 DAA. Para análise de controle das plantas daninhas foram utilizadas faixas laterais (bordaduras), dispostas paralelamente aos blocos da área experimental. Entretanto, a partir dos 28 DAA, assim como o tratamento testemunha, todos os demais foram capinados manualmente e mantidos sem infestação durante o restante do ciclo da cultura.

Os dados obtidos foram submetidos à análise de variância pelo teste $\mathrm{F}$, e as suas médias, comparadas pelo teste t a $5 \%$ de probabilidade. A escolha do teste t como um teste menos rigoroso e mais adequado a estudos de seletividade de herbicidas foi feita seguindo as sugestões de Azania et al. (2005) e Carvalho et al. (2009).

\section{RESULTADOS E DISCUSSÃO}

Os tratamentos estudados apresentaram alta seletividade para mamoneira AL Guarany 2002, sendo apenas identificados baixos níveis de fitointoxicação visual aos 7 DAA, com exceção dos herbicidas tepraloxydim e isoxaflutole, em que os valores foram de 12,5 e 70,7\%, respectivamente (Tabela 1). Os resultados demonstram aos 14 DAA ausência de qualquer sintoma visivel de injúria e/ou danos na parte aérea da variedade AL Guarany 2002 
Tabela 1 - Fitointoxicação (\%) da mamoneira submetida à aplicação em pós-emergência de herbicidas graminicidas aos 7, 14, 21 e 28 dias após aplicação (DAA)

\begin{tabular}{|c|c|c|c|c|c|}
\hline \multirow[b]{2}{*}{ Tratamento } & \multirow{2}{*}{\begin{tabular}{|c|} 
Dosagem de herbicida \\
$\begin{array}{c}\left(\mathrm{g} \mathrm{ha}^{-1}\right)+\text { Adjuvante } \\
(\% \mathrm{v} / \mathrm{v})\end{array}$
\end{tabular}} & \multicolumn{4}{|c|}{ Fitointoxicação (\%) } \\
\hline & & 7 DAA & 14 DAA & $21 \mathrm{DAA}$ & 28 DAA \\
\hline Fluazifop-p-butyl & $313+0,2^{\frac{1}{}}$ & $0,5 \mathrm{a}$ & $0,0 \mathrm{a}$ & $0,0 \mathrm{a}$ & 0,0 \\
\hline Sethoxydim & $322+0,5^{2 /}$ & $1,5 \mathrm{a}$ & $0,0 \mathrm{a}$ & $0,0 \mathrm{a}$ & 0,0 \\
\hline Haloxyfop-methyl & $120+0,5^{\frac{2}{}}$ & $1,7 \mathrm{a}$ & $0,0 \mathrm{a}$ & $0,0 \mathrm{a}$ & 0,0 \\
\hline Clethodim+fenoxaprop-p-ethyl & $75+75+0,5^{2 \prime}$ & $2,5 \mathrm{a}$ & $0,7 \mathrm{a}$ & $0,0 \mathrm{a}$ & 0,0 \\
\hline Quizalofop-p-ethyl & $125+0,5^{2 /}$ & $1,2 \mathrm{a}$ & $0,0 \mathrm{a}$ & $0,0 \mathrm{a}$ & 0,0 \\
\hline Clethodim & $156+0,5^{2 /}$ & $1,2 \mathrm{a}$ & $0,0 \mathrm{a}$ & $0,0 \mathrm{a}$ & 0,0 \\
\hline Propaquizafop & $175+0,5^{2 /}$ & $0,7 \mathrm{a}$ & $0,0 \mathrm{a}$ & $0,0 \mathrm{a}$ & 0,0 \\
\hline Tepraloxydim & $400+0,5^{3 /}$ & $12,5 \mathrm{~b}$ & $10,7 \mathrm{~b}$ & $4,0 \mathrm{~b}$ & 0,0 \\
\hline Butroxydim & $100+0,5^{3 /}$ & $3,0 \mathrm{a}$ & $0,0 \mathrm{a}$ & $0,0 \mathrm{a}$ & 0,0 \\
\hline Isoxaflutole & 60 & $70,7 \mathrm{c}$ & $37,0 \mathrm{c}$ & $16,2 \mathrm{c}$ & 10,7 \\
\hline Testemunha capinada & - & $0,0 \mathrm{a}$ & $0,0 \mathrm{a}$ & $0,0 \mathrm{a}$ & 0,0 \\
\hline F & - & $187,25^{*}$ & $127,76^{*}$ & $147,35^{*}$ & - \\
\hline CV $(\%)$ & - & 35,0 & 45,25 & 44,10 & - \\
\hline DMS $(5 \%)$ & - & 4,40 & 2,88 & 1,17 & - \\
\hline
\end{tabular}

${ }^{1 /}$ Agral $^{\oplus ;}{ }^{2 /}$ Assist $^{\oplus} ;{ }^{3 /}$ Dash $^{\circledast}$. Valores seguidos de mesma letra na mesma coluna não diferem entre si pelo teste de $\mathrm{t}$ a $5 \%$ de probabilidade $*=\mathrm{p} \leq 0,05 ;{ }^{\mathrm{NS}}=$ não significativo.

para aplicação dos herbicidas inibidores de acetil coenzima-A carboxilase (ACCase), com exceção de tepraloxydim $(10,7 \%)$ e isoxaflutole $(37,0 \%)$, sendo este último um inibidor da biossintese de carotenos (HPPD), de acordo com a Figura 1. Aos 21 DAA, e principalmente 28 DAA, apenas para o isoxaflutole ainda persistiram injúrias visiveis de fitointoxicação $(10,7 \%)$, representadas por manchas cloróticas e necrosamento, irregularmente distribuídas nas folhas mais velhas da cultura. Segundo Vidal et al. (2000), a grande seletividade dos herbicidas inibidores de ACCase às espécies dicotiledôneas em geral explica-se pelo tipo e pela compartimentalização dessa enzima na célula. Entretanto, ainda assim, esses autores afirmaram existir variabilidade entre espécies da mesma família das Cucurbitaceae na sensibilidade ao herbicida fluazifop-p-butil.

Com relação ao teor de clorofila das folhas mais novas, localizadas no terço superior das plantas da mamoneira AL Guarany 2002, novamente não foram verificadas diferenças significativas entre os herbicidas inibidores de ACCase e a testemunha, ao contrário do isoxaflutole, que se apresentou significativamente inferior à testemunha e aos inibidores de ACCase aos 7 e 21 DAA, corroborando os resultados de fitointoxicação visual (Tabela 2). No que se refere às folhas localizadas no terço inferior das plantas, aos 7 e 21 DAA não foram identificadas diferenças significativas entre os tratamentos testados.

O comprimento do caule da mamoneira não foi influenciado pelos tratamentos herbicidas (Tabela 3), ao contrário do diâmetro caulinar aos 7 DAA, em que a testemunha capinada apenas diferiu significativamente do tetraloxydim, sendo, em termos médios, 20,5\% inferior. Aos 21 DAA, apenas o isoxaflutole apresentou-se com diâmetro caulinar significativamente inferior ao dos demais tratamentos que se igualaram à testemunha, e o tepraloxydim manteve-se superior à testemunha aos 7 e 21 DAA. Esse aumento de espessura do caule sem prejudicar o seu comprimento, verificado apenas para o tepraloxydim, pode ter sido uma resposta de desintoxicação e/ou metabolização, uma vez que foi o único inibidor de ACCase onde foram identificadas injúrias na parte aérea da mamoneira até os 21 DAA. Esses resultados corroboram os citados por Maciel et al. (2007), onde foi constatada redução do diâmetro 

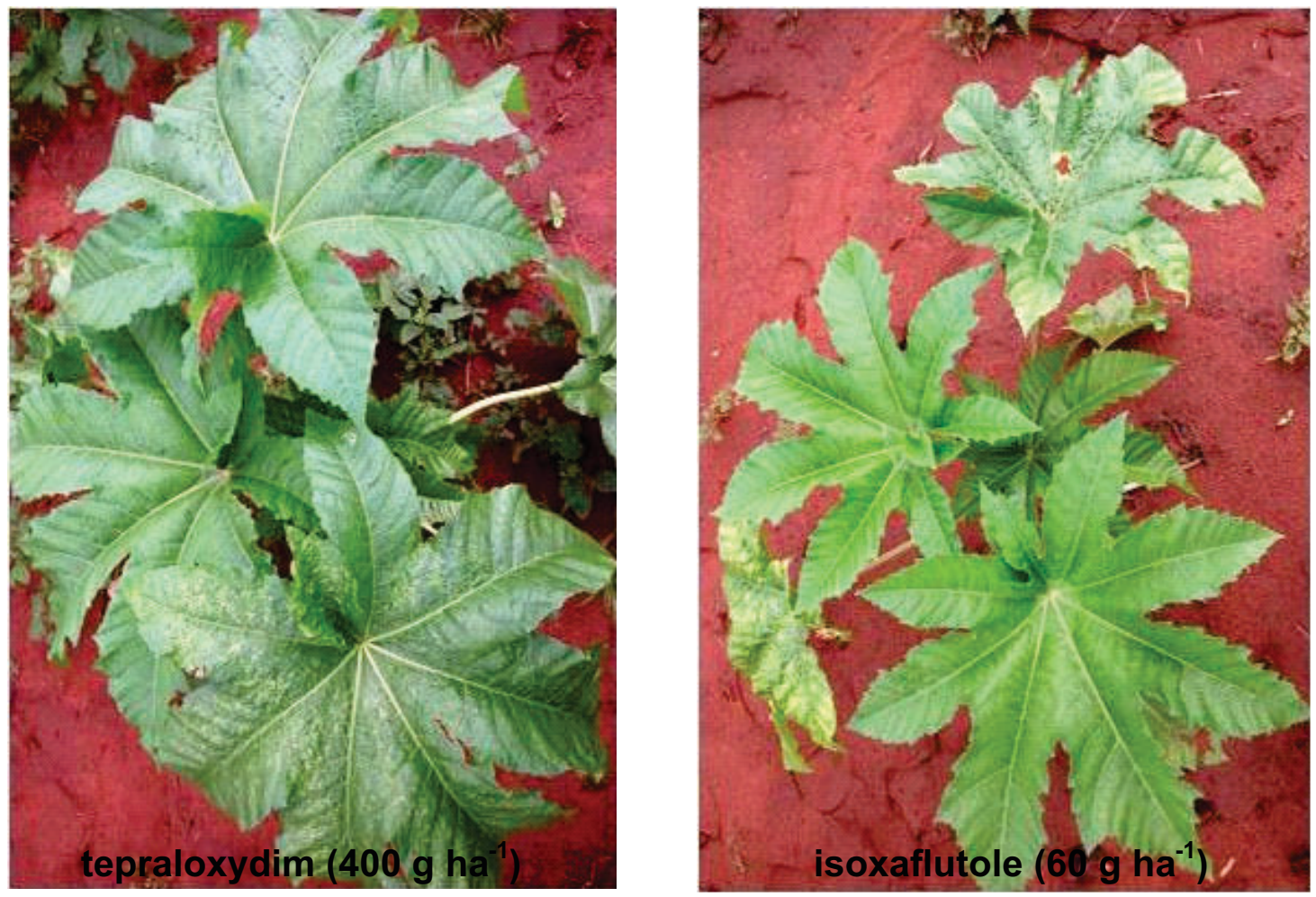

Figura 1 - Caracterização dos sintomas de fitointoxicação na variedade de mamoneira AL Guarany 2002 aos 14 DAA, provocados pela aplicação em pós-emergência de tepraloxydim (inibidor de ACCase) e isoxaflutole (inibidor da síntese HPPD).

Tabela 2 - Teor de clorofila nas folhas da mamoneira submetida à aplicação em pós-emergência de herbicidas graminicidas aos 7 e $21 \mathrm{DAA}$

\begin{tabular}{|c|c|c|c|c|c|}
\hline \multirow{3}{*}{ Tratamento } & \multirow{3}{*}{$\begin{array}{l}\text { Dosagem herbicida } \\
\left(\mathrm{g} \mathrm{ha}^{-1}\right)+ \\
\text { Adjuvante }(\% \mathrm{v} / \mathrm{v})\end{array}$} & \multicolumn{4}{|c|}{ Teor de clorofila $\left(\mu \mathrm{g} \mathrm{dm}^{-2}\right)$} \\
\hline & & \multicolumn{2}{|c|}{ (Terço superior) } & \multicolumn{2}{|c|}{ (Terço inferior) } \\
\hline & & $7 \mathrm{DAA}$ & $21 \mathrm{DAA}$ & 7 DAA & $21 \mathrm{DAA}$ \\
\hline Fluazifop-p-butyl & $313+0,2^{\frac{1 /}{\prime}}$ & $38,25 \mathrm{a}$ & $45,62 \mathrm{a}$ & 40,25 & 48,12 \\
\hline Sethoxydim & $322+0,5^{2 /}$ & $41,00 \mathrm{a}$ & $48,75 \mathrm{a}$ & 45,50 & 49,75 \\
\hline Haloxyfop-methyl & $120+0,5^{2 !}$ & $39,75 \mathrm{a}$ & $48,12 \mathrm{a}$ & 40,75 & 49,60 \\
\hline Clethodim+fenoxaprop-p-ethyl & $75+75+0,5^{2^{\prime}}$ & $40,00 \mathrm{a}$ & $46,00 \mathrm{a}$ & 43,50 & 49,75 \\
\hline Quizalofop-p-ethyl & $125+0,5^{2 /}$ & $39,50 \mathrm{a}$ & $46,12 \mathrm{a}$ & 40,75 & 48,12 \\
\hline Clethodim & $156+0,5^{2 /}$ & $41,25 \mathrm{a}$ & $45,50 \mathrm{a}$ & 45,25 & 49,87 \\
\hline Propaquizafop & $175+0,5^{2 /}$ & $39,50 \mathrm{a}$ & $48,00 \mathrm{a}$ & 41,75 & 49,62 \\
\hline Tepraloxydim & $400+0,5^{3^{\prime}}$ & $40,00 \mathrm{a}$ & $46,37 \mathrm{a}$ & 41,75 & 49,75 \\
\hline Butroxydim & $100+0,5^{\frac{3}{}}$ & $40,75 \mathrm{a}$ & $46,12 \mathrm{a}$ & 42,25 & 48,75 \\
\hline Isoxaflutole & 60 & $31,75 \mathrm{~b}$ & $38,00 \mathrm{~b}$ & 40,25 & 47,37 \\
\hline Testemunha capinada & - & $40,00 \mathrm{a}$ & $49,12 \mathrm{a}$ & 41,50 & 49,50 \\
\hline$F$ & - & $5,98^{*}$ & $3,65^{*}$ & $0,63^{\mathrm{NS}}$ & $0,28^{\mathrm{NS}}$ \\
\hline $\mathrm{CV}(\%)$ & - & 5,46 & 6,81 & 11,12 & 6,63 \\
\hline DMS $(5 \%)$ & - & 3,09 & 4,54 & 6,77 & 4,70 \\
\hline
\end{tabular}

${ }^{1 /}$ Agral $^{\circledR} ;{ }^{2} /$ Assist $^{\circledR} ;{ }^{3 /}$ Dash $^{\circledR}$. Valores seguidos de mesma letra na mesma coluna não diferem entre si pelo teste $t$ a $5 \%$ de probabilidade. $*=\mathrm{p} \leq 0,05 ;{ }^{N S}=$ não significativo. 
Tabela 3 - Comprimento e diâmetro mediano de caule da mamoneira submetida à aplicação em pós-emergência de herbicidas graminicidas aos 7 e 21 DAA

\begin{tabular}{|c|c|c|c|c|c|}
\hline \multirow[b]{2}{*}{ Tratamento } & \multirow{2}{*}{$\begin{array}{c}\text { Dosagem herbicida } \\
\begin{array}{c}\left(\mathrm{g} \mathrm{ha}^{-1}\right)+\text { Adjuvante } \\
(\% \mathrm{v} / \mathrm{v})\end{array}\end{array}$} & \multicolumn{2}{|c|}{ Comprimento do caule $(\mathrm{cm})$} & \multicolumn{2}{|c|}{ Diâmetro do caule $(\mathrm{mm})$} \\
\hline & & $7 \mathrm{DAA}$ & $21 \mathrm{DAA}$ & $7 \mathrm{DAA}$ & $21 \mathrm{DAA}$ \\
\hline Fluazifop-p-butyl & $313+0,2^{\frac{1}{}}$ & 23,25 & 37,37 & $13,57 \mathrm{~cd}$ & $26,37 \mathrm{ab}$ \\
\hline Sethoxydim & $322+0,5^{2 /}$ & 25,12 & 41,12 & $16,25 \mathrm{ab}$ & $26,37 \mathrm{ab}$ \\
\hline Haloxyfop-methyl & $120+0,5^{2^{\prime}}$ & 23,37 & 40,00 & $17,62 \mathrm{ab}$ & $26,37 \mathrm{ab}$ \\
\hline Clethodim+fenoxaprop-p-ethyl & $75+75+0,5^{\frac{2}{}}$ & 25,87 & 39,00 & $16,00 \mathrm{abc}$ & $26,25 \mathrm{ab}$ \\
\hline Quizalofop-p-ethyl & $125+0,5^{2 /}$ & 24,25 & 36,00 & $15,87 \mathrm{bc}$ & $26,62 \mathrm{ab}$ \\
\hline Clethodim & $156+0,5^{2 /}$ & 24,25 & 35,62 & $16,50 \mathrm{ab}$ & $27,87 \mathrm{ab}$ \\
\hline Propaquizafop & $175+0,5^{2^{\prime}}$ & 25,25 & 40,62 & $16,12 \mathrm{ab}$ & $27,37 \mathrm{ab}$ \\
\hline Tepraloxydim & $400+0,5^{\frac{3}{}}$ & 23,50 & 42,25 & $18,37 \mathrm{a}$ & $29,00 \mathrm{a}$ \\
\hline Butroxydim & $100+0,5^{\frac{3}{}}$ & 23,00 & 33,25 & $13,25 \mathrm{~d}$ & $26,12 \mathrm{ab}$ \\
\hline Isoxaflutole & 60 & 22,25 & 33,50 & $13,00 \mathrm{~d}$ & $20,00 \mathrm{c}$ \\
\hline Testemunha capinada & - & 21,25 & 36,25 & $15,25 \mathrm{bcd}$ & $24,00 \mathrm{bc}$ \\
\hline$F$ & - & $0,63^{\mathrm{N} \overline{\mathrm{S}}}$ & $0,88^{\mathrm{NS}}$ & $4,21^{*}$ & $2,71^{*}$ \\
\hline $\mathrm{CV}(\%)$ & - & 14,48 & 16,89 & 10,82 & 10,96 \\
\hline DMS $(5 \%)$ & - & 4,97 & 9,18 & 2,13 & 0,75 \\
\hline
\end{tabular}

${ }^{1 /}$ Agral $^{\oplus ;}{ }^{2 /}$ Assist $^{\oplus} ;{ }^{3 /}$ Dash $^{\circledast}$. Valores seguidos de mesma letra na mesma coluna não diferem entre si pelo teste $\mathrm{t}$ a $5 \%$ de probabilidade. ${ }^{*}$ $=\mathrm{p} \leq 0,05 ;$ NS $=$ não significativo.

mediano de caule para os herbicidas alachlor (inibidor de crescimento da parte aérea), pendimethalin e trifluralin (inibidores da polimerização de tubulina), aplicados em préemergência isolados e/ou em mistura entre si, sem interferir na altura da variedade $\mathrm{AL}$ Guarany 2002.

Com relação à produtividade, todos os herbicidas inibidores de ACCase apresentaram-se altamente seletivos, não diferindo significativamente em relação à testemunha capinada, ao contrário do observado para o isoxaflutole, em que a redução em relação à testemunha foi, em média, da ordem de $31 \%$ (Figura 2). Tomando por base a variação entre 1.680 e $1.950 \mathrm{~kg} \mathrm{ha}^{-1}$ da produtividade de cultivares do Instituto Agronômico de Campinas (IAC) nas safras 1999/2000, 2002/2003, 2003/ 2004 e 2006, em três municípios paulistas (Savy Filho et al., 2007), observou-se que as médias alcançadas na testemunha capinada $\left(1.229 \mathrm{~kg} \mathrm{ha}^{-1}\right)$ e com os herbicidas inibidores de ACCase (1.332 $\left.\mathrm{kg} \mathrm{ha}^{-1}\right)$ ficaram na mesma faixa prevista para o Estado de São Paulo.

Todos os herbicidas controlaram C. echinatus, com níveis de eficácia superiores a 95\%, principalmente a partir dos 21 DAA (Tabela 4). Entretanto, foi possivel constatar, desde os $7 \mathrm{DAA}$, que o sethoxydim e isoxaflutole, assim como o quizalofop-p-ethyl, foram os que apresentaram menor velocidade de controle de C. echinatus; apesar de eles terem atingido níveis satisfatórios aos 14 DAA, diferiram significativamente dos demais herbicidas, por apresentarem eficácia abaixo de $95 \%$. Aos 28 DAA, o isoxaflutole e o quizalofop-p-ethyl, mesmo promovendo excelente de controle de C. echinatus $(98,7$ e 99,2\%), ainda diferiram significativamente dos demais tratamentos. Outros resultados de controle eficiente de $C$. echinatus foram relatados por Cruz et al. (1991) e Barroso et al. (2010), utilizando-se, respectivamente, fluazifop-p-butil (187 $\mathrm{g} \mathrm{ha}^{-1}$ ) e haloxyfop-methyl (240 $\mathrm{g} \mathrm{ha}^{-1}$ ) na cultura do amendoim e haloxyfop-methyl (60 $\left.\mathrm{g} \mathrm{ha}^{-1}\right)$ e tepraloxydim (100 $\mathrm{g} \mathrm{ha}^{-1}$ ) na cultura da soja.

É importante ressaltar que, apesar de a mamoneira AL Guarany 2002 ter apresentado alta seletividade aos herbicidas inibidores de ACCase mesmo para doses superiores às normalmente utilizadas em outras culturas oleaginosas, ainda assim faz-se necessário o 
conhecimento técnico por parte dos extensionistas e agricultores sobre o comportamento dessa classe de herbicidas para as espécies de gramíneas invasoras prevalecentes na cultura, para escolha da opção mais adequada da ferramenta de manejo.

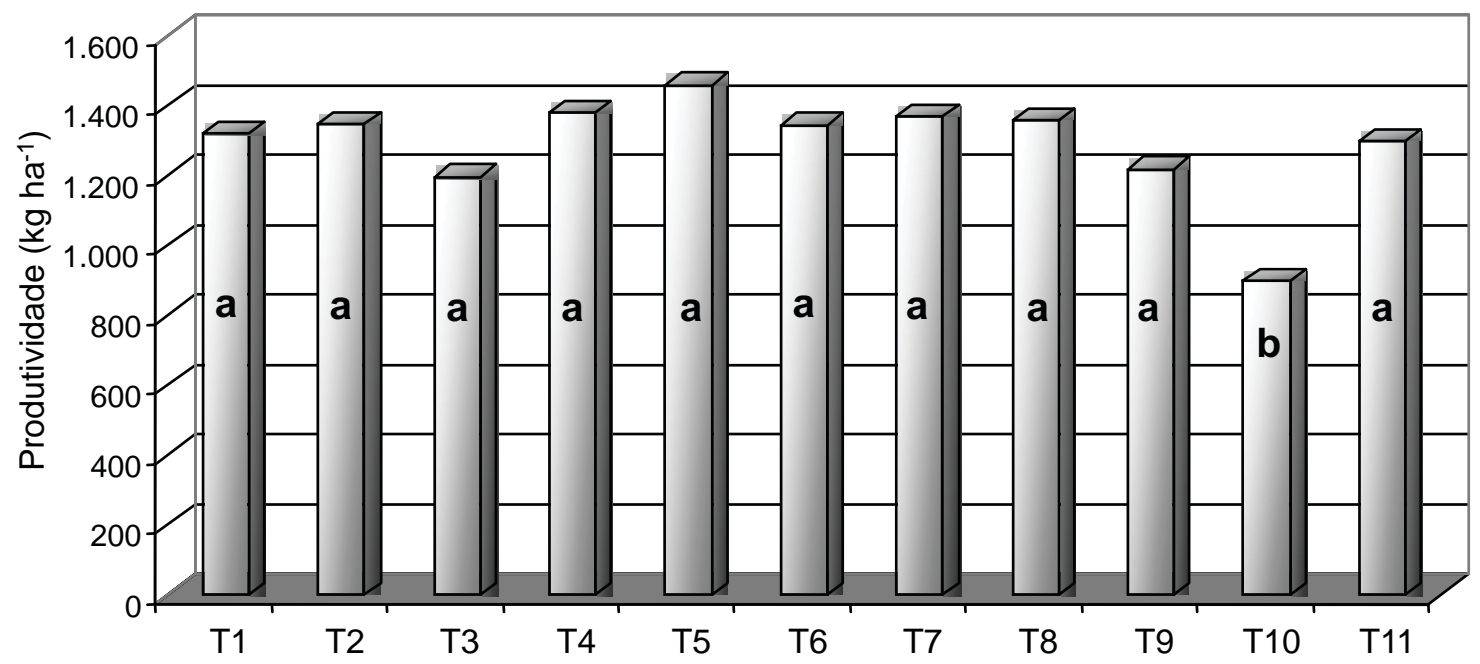

(T1) fluazifop-p-butyl + Agral $^{\circledR}\left(313 \mathrm{~g} \mathrm{ha}^{-1}+0,2 \%\right)$; (T2) sethoxydim + Assist $^{\circledR}\left(322 \mathrm{~g} \mathrm{ha}^{-1}+0,5 \%\right) ;+(\mathrm{T} 3)$ haloxyfop-methyl + Assist ${ }^{\circledR}$ (120 $\left.\mathrm{g} \mathrm{ha}^{-1}+0,5 \%\right)$; (T4) clethodim+fenoxaprop-p-ethyl + Assist ${ }^{\circledR}\left(75+75 \mathrm{~g} \mathrm{ha}^{-1}+0,5 \%\right)$; (T5) quizalofop-p-ethyl + Assist ${ }^{\circledR}\left(125 \mathrm{~g} \mathrm{ha}^{-1}+\right.$ $0,5 \%)$; (T6) clethodim + Assist $^{\circledR}\left(156 \mathrm{~g} \mathrm{ha}^{-1}+0,5 \%\right)$; (T7) propaquizafop + Assist $^{\circledast}\left(175 \mathrm{~g} \mathrm{ha}^{-1}+0,5 \%\right)$; (T8) tepraloxydim + Dash ${ }^{\circledR}$ $\left(400 \mathrm{~g} \mathrm{ha}^{-1}+0,5 \%\right)$; (T9) butroxydim $+\operatorname{Dash}^{\circledast}\left(100 \mathrm{~g} \mathrm{ha}^{-1}+0,5 \%\right)$; (T10) isoxaflutole $\left(60 \mathrm{~g} \mathrm{ha}^{-1}\right)$ e (T11) testemunha capinada. Valores seguidos de mesma letra não diferem entre si pelo teste t a $5 \%$ de probabilidade.

Figura 2 - Produtividade da variedade de mamoneira AL Guarany 2002 submetida à aplicação em pós-emergência de herbicidas graminicidas.

Tabela 4 - Porcentagem de controle de Cenchrus echinatus na cultura da mamoneira submetida à aplicação em pós-emergência de herbicidas graminicidas aos 7, 14, 21 e 28 DAA

\begin{tabular}{|c|c|c|c|c|c|}
\hline \multirow{2}{*}{ Tratamento } & \multirow{2}{*}{$\begin{array}{c}\text { Dosagem herbicida } \\
\left(\mathrm{g} \mathrm{ha}^{-1}\right)+ \\
\text { Adjuvante }(\% \mathrm{v} / \mathrm{v})\end{array}$} & \multicolumn{4}{|c|}{ Cenchrus echinatus $(\%)$} \\
\hline & & 7 DAA & 14 DAA & $21 \mathrm{DAA}$ & 28 DAA \\
\hline Fluazifop-p-butyl & $313+0,2^{\frac{1 /}{1}}$ & $89,5 \mathrm{ab}$ & $96,5 \mathrm{ab}$ & $99,5 \mathrm{ab}$ & $100,0 \mathrm{a}$ \\
\hline Sethoxydim & $322+0,5^{2 /}$ & $72,0 \mathrm{c}$ & $82,5 \mathrm{~d}$ & $95,0 \mathrm{~d}$ & $99,5 \mathrm{ab}$ \\
\hline Haloxyfop-methyl & $120+0,5^{2 /}$ & $90,0 \mathrm{ab}$ & $98,2 \mathrm{a}$ & $95,5 \mathrm{ab}$ & $100,0 \mathrm{a}$ \\
\hline Clethodim+fenoxaprop-p-ethyl & $75+75+0,5^{\frac{2}{}}$ & $88,2 \mathrm{ab}$ & $95,7 \mathrm{ab}$ & $95,5 \mathrm{ab}$ & $100,0 \mathrm{a}$ \\
\hline Quizalofop-p-ethyl & $125+0,5^{\frac{2}{}}$ & $83,7 \mathrm{~b}$ & $92,7 \mathrm{bc}$ & $97,5 \mathrm{bc}$ & $99,2 \mathrm{bc}$ \\
\hline Clethodim & $156+0,5^{2 /}$ & $87,5 \mathrm{ab}$ & $97,2 \mathrm{a}$ & $99,2 \mathrm{ab}$ & $99,5 \mathrm{ab}$ \\
\hline Propaquizafop & $175+0,5^{2 /}$ & $94,5 \mathrm{a}$ & $97,2 \mathrm{a}$ & $98,7 \mathrm{ab}$ & $99,5 \mathrm{ab}$ \\
\hline Tepraloxydim & $400+0,5^{3^{3 /}}$ & $90,5 \mathrm{ab}$ & $95,5 \mathrm{ab}$ & $100,0 \mathrm{a}$ & $100,0 \mathrm{a}$ \\
\hline Butroxydim & $100+0,5^{\frac{3}{}}$ & $90,0 \mathrm{ab}$ & $96,2 \mathrm{ab}$ & $100,0 \mathrm{a}$ & $100,0 \mathrm{a}$ \\
\hline Isoxaflutole & 60 & $70,0 \mathrm{c}$ & $89,5 \mathrm{c}$ & $96,2 \mathrm{~cd}$ & $98,7 \mathrm{c}$ \\
\hline Testemunha capinada & - & $0,0 \mathrm{~d}$ & $0,0 \mathrm{e}$ & $0,0 \mathrm{e}$ & $0,0 \mathrm{~d}$ \\
\hline $\mathrm{F}$ & - & $83,1^{*}$ & $432,3^{*}$ & $1.631,8^{*}$ & $1.3467,0^{*}$ \\
\hline $\mathrm{CV}(\%)$ & - & 7,60 & 3,23 & 1,64 & 0,57 \\
\hline DMS $(5 \%)$ & - & 8,54 & 3,99 & 2,13 & 0,75 \\
\hline
\end{tabular}

${ }^{1 /}$ Agral $^{\circledR} ;{ }^{2 /}$ Assist $^{\circledR} ;{ }^{3 /}$ Dash $^{\circledast}$. Valores seguidos de mesma letra na mesma coluna não diferem entre si pelo teste $\mathrm{t}$ a $5 \%$ de probabilidade. $*=\mathrm{p} \leq 0,05 ;$ NS $=$ não significativo. 
Entretanto, em função do elevado espectro de ação dos herbicidas inibidores de ACCase sobre as principais espécies de gramíneas anuais (Rodrigues \& Almeida, 2005) e da seletividade da cultura da mamoneira AL Guarany 2002 verificada no trabalho, as recomendações com produtos desse mecanismo de ação podem ser mais seguras quanto à escolha de produto e doses, principalmente em situações semelhantes às descritas por Barroso et al. (2010), em que não se dispõe de especialistas para correta identificação da infestação.

A mamoneira cv. AL Guarany 2002 apresentou alta seletividade aos herbicidas inibidores de ACCase, com exceção do tepraloxydim, onde os sintomas persistiram até os 21 DAA, e do isoxaflutole (inibidor de HPPD), por apresentar injúrias nas folhas mais velhas e redução significativa de produtividade. A infestação de C. echinatus foi eficientemente controlada pelos herbicidas inibidores de ACCase entre 14 e 21 DAA ( $\geq 95,0 \%$ ).

\section{LITERATURA CITADA}

AZANIA, C. A. M. et al. Seletividade de herbicidas. I utilização do método de testemunhas pareadas em experimento com cana-de-açúcar. Planta Daninha, v. 23, n. 4, p. 661-667, 2005.

BARROSO, A. L. L. et al. Eficácia de herbicidas inibidores da ACCase no controle de gramíneas em lavouras de soja. Planta Daninha, v. 28, n. 1, p. 149-157, 2010.

CARVALHO, S. J. P. et al. Herbicide selectivity by differential metabolism: considerations for reducing crop damages. Sci. Agríc., v. 66, n. 1, p. 136-142, 2009.

\section{COMPANHIA NACIONAL DE ABASTECIMENTO -} CONAB. Conjuntura Mensal: Mamona Setembro 2010 Disponível em: <http://www.conab.gov.br/OlalaCMS/ uploads/arquivos/91cad7d1ff4494bc89582e87b82aa5e9.pdf >. Acesso em: 25 out. 2010.

CRUZ, L. S. P. et al. Herbicidas de aplicação em pósemergência em amendoim: I. Controle de plantas daninhas e persistência no solo. Bragantia, v. 50, n. 1, p. 103-114, 1991
FAO - STATISTICS. Production and trade - Castor beans, 2007. Disponível em: $<$ http://faostat.fao.org $>$. Acesso em: 07 jul. 2009

MACIEL, C. D. G. et al. Seletividade de herbicidas em cultivares de mamona. R. Bras. Oleag. Fibr., v. 11, n. 1, p. $47-54,2007$

MACIEL, C. D. G. et al. Possibilidade de aplicação de misturas de herbicidas de ação total com jato dirigido em mamoneira de porte anão. Planta Daninha, v. 26, n. 2, p. 457-464, 2008.

MELHORANÇA, A. L.; STAUT, L. A. Indicações técnicas para a cultura da mamona no Mato Grosso do Sul. Dourados: Embrapa Agropecuária Oeste, 2005. 65 p.

OLIVEIRA, I. P. et al. R. Eletrônica Faculdade Montes Belos, v. 1, n. 2, p. 104-130, 2005.

RODRIGUES, B. N.; ALMEIDA, F. S. Guia de herbicidas. 5.ed. Londrina: Edição dos Autores, 2005. 591 p.

SAVY FILHO, A. Mamona: tecnologia agrícola. Campinas: EMOPI, 2005. 105 p

SAVY FILHO, A. et al. IAC-2028: nova cultivar de mamona Pesq. Agropec. Bras., v. 42, n. 3, p. 449-452, 2007.

SEVERINO, L. S. et al. Crescimento e produtividade da mamoneira sob fertilização química em região semiárida. Campina Grande: Embrapa Algodão, 2006. 19 p. (Boletim de Pesquisa e Desenvolvimento, 62)

SILVA, T. R. B. et al. Adubação nitrogenada em cobertura na cultura da mamona em plantio direto. Pesq. Agropec. Bras., v. 42, n. 9, p. 1357-1359, 2007.

SOCIEDADE BRASILEIRA DA CIÊNCIA DAS PLANTAS DANINHAS - SBCPD. Procedimentos para instalação, avaliação e análise de experimentos com herbicidas. Londrina: $1995.42 \mathrm{p}$.

VIDAL, R. A. et al. Seletividade do herbicida fluazifop-pbutil para cucurbitáceas. Planta Daninha, v. 18, n. 3, p. 413-417, 2000.

VIDAL, R. A.; MEROTTO Jr., A. Herbicidologia. Porto Alegre: Evangraf, 2001. 152 p. 